
\title{
$\begin{array}{ll}\text { Research Square } & \begin{array}{l}\text { Preprints are preliminary reports that have not undergone peer review. } \\ \text { They should not be considered conclusive, used to inform clinical practice, } \\ \text { or referenced by the media as validated information. }\end{array}\end{array}$
}

\section{Can prolong life with nine turn method (Yan Nian Jiu Zhuan) Qigong alleviates Fatigue, Sleep quality, Depression and anxiety on Patients with Chronic Fatigue Syndrome: a Randomized, Controlled, Clinical Study?}

\author{
Fangfang Xie \\ Shanghai University of TCM: Shanghai University of Traditional Chinese Medicine \\ Jiatuo Xu \\ Shanghai University of TCM: Shanghai University of Traditional Chinese Medicine \\ Chong Guan \\ Shanghai University of TCM: Shanghai University of Traditional Chinese Medicine \\ Ziji Cheng \\ Shanghai University of TCM: Shanghai University of Traditional Chinese Medicine \\ Yuanjia Gu \\ Shanghai University of TCM: Shanghai University of Traditional Chinese Medicine \\ Ziying Chen \\ Shanghai University of TCM: Shanghai University of Traditional Chinese Medicine \\ Chaoqun Xie \\ Shanghai University of TCM: Shanghai University of Traditional Chinese Medicine \\ Jue Wang \\ Shanghai University of TCM: Shanghai University of Traditional Chinese Medicine \\ Yuling Shi \\ Shanghai University of TCM: Shanghai University of Traditional Chinese Medicine \\ XuXiang Ma \\ Shanghai University of TCM: Shanghai University of Traditional Chinese Medicine \\ Tao Jiang \\ Shanghai University of TCM: Shanghai University of Traditional Chinese Medicine \\ Fei Yao ( $\nabla$ doctoryaofei@126.com) \\ Shanghai University of TCM https://orcid.org/0000-0002-6055-1336 \\ Yanli You \\ Changhai Hospital
}

\section{Research}

Keywords: fatigue, treatment, sleep, qigong, anxiety

Posted Date: October 28th, 2021

DOl: https://doi.org/10.21203/rs.3.rs-965010/v1

License: (9) (7) This work is licensed under a Creative Commons Attribution 4.0 International License. Read Full License 


\section{Abstract \\ Background}

Chronic fatigue syndrome (CFS) is a complex disease with unknown etiology and mechanism. Prolong life with nine turn method (PLWNT) Qigong is a system of mind-body exercise with restorative benefits that have shown a wide range of benefits in the treatment of CFS. The purpose of this study is to investigate the effect of PLWNT Qigong exercise on CFS with a focus on fatigue, sleep quality, depression and anxiety.

\section{Methods}

A total of 135 participants were randomly divided into treatment groups and control group in the same proportion. The treatment groups received PLWNT Qigong exercise (PLWNT Group) or cognitive behavioral therapy treatment (CBT Group). The healthy control group (HC Group) does not receive any treatment and maintains daily life. Participants from treatment groups were taught by related highly qualified professor at the Shanghai University of Traditional Chinese Medicine once a week and were supervised online during the remaining 6 days at home, over 12 consecutive weeks. The primary outcome was fatigue (MFI20 ) and secondary outcomes were sleep quality (PSQI), anxiety and depression (HADS). All groups were measured at baseline ( $\left.T_{0}\right)$ and post-intervention ( $\left.T_{1}\right)$.

\section{Results}

134 patient completed the study (45 in the PLWNT Group, 44 in the CBT Group and 45 in HC Group). The within group comparison of PLWNT and CBT groups were all showed significant improvement in MFI-20, PSQI and HADS after the intervention (p凶0.05). No significant difference was found between the PLWNT group and CBT group(pه0.05),but the total effective rate of the PLWNT group was $62.22 \%$, which was higher than the CBT group was $50.00 \%$. The changes in the PLWNT group and CBT group were all higher than those in the HC group except for habitual sleep efficiency and sleep medicine using(p囚0.05). In additional, fatigue degree changes in the PLWBT group were positively correlated with sleep degree $(r=0.315)$ and anxiety degree( $r=0.333)$, but only anxiety degree $(r=0.332)$ was found to be positively correlated with fatigue in the CBT group.

\section{Conclusion}

PLWNT Qigong exercise has the potential as a rehabilitation method on CFS with a focus on fatigue, sleep quality, anxiety and depression. Yet, the results need to be interpreted carefully and needs to be repeated in a larger sample. Future studies will expand the sample size for further in-depth research to determine the frequency and intensity of PLWNT Qigong intervention that is particularly beneficial to CFS. The study registered in the American Clinical Trial Registry on 4 November 2018, the registration number is NCT03496961.

\section{Introduction}

Chronic fatigue syndrome (CFS) is a debilitating disease which is poorly understood (1). It is commonly characterized by chronic fatigue lasting more than 6 months without alleviation after resting, accompanied by cognitive dysfunction, sleep problems, autonomic dysfunction, post-exertional malaise, severely impaired activities of daily living, and/or pain in muscles or joints(2). According to worldwide statistics, approximately $1 \%$ of the worldwide population, 17 to 24 million people suffer from CFS with a significant incidence of fatigue, psychiatric comorbidities and cognitive symptoms(3). The patients of CFS are forced to reduce $50 \%$ of their daily activities (4) and $87-95 \%$ of them have related daytime dysfunction $(5,6)$. A large sample of 1409 CFS study showed that $95 \%$ of people with higher education and full-time work had sleep disorders, $68-80 \%$ suffered from anxiety and depression symptoms (7), which further incurs the decline in life quality $(8,9)$. In addition, fatigue can occur independently of other symptoms of CFS, but it was usually related to sleep and mood disorders $(10$, 11). A study reported that fatigue has a negative impact on all areas of life quality, including physical and emotional health, activity ability, and activities of daytime living(12). The increasing incidence of CFS, not only threatens the personal wellbeing of more people, but also brings a heavy burden to families and the society.

Since the etiology and pathology of CFS are unknown, symptomatic treatments are used clinically, including drugs, exercise therapy, cognitive behavioral therapy (CBT), which can relieve CFS symptoms and improve life quality. The latest CFS treatment guidelines emphasize the change in the overall treatment attitude of CFS, for instance, drug treatment is now regarded as an adjuvant treatment of CFS non-pharmacological intervention(13). Several researchers propose that graded exercise therapy and CBT might be effective efficacious treatments for CFS to improve fatigue and poor mental health, including depression, anxiety and schizophrenia $(14,15)$. However, evidences of persistent and sustained significant outcomes in CFS patients are not sufficient(16). The clinical manual published by International Association of Chronic Fatigue Syndrome (IACFS) recommends traditional Chinese medicine (TCM) treatments including acupuncture and massage.

Qigong (pronounced "chee gun") is one of the traditional Chinese medicine methods that has been used for thousands of years to optimize and restore the energy of the body, mind, and spirit(17). It is a combination of asanas, relaxation, breathing regulation, meditation, and gentle exercise, designed to improve overall health and promote the integration of body and mind $(18,19)$. Specifically, the term "Qigong" involves two theories: "Qi", that is, the righteous qi of the body; "Gong", that is the training or cultivation of qi. Qigong can be practiced both in a static (sitting, lying, standing) and a dynamic (moving) way. Its purpose is to achieve the flow of qi and blood $(20,21)$. Qi is the vital energy flowing in the meridians of the body, and long-term practice of Qigong helps to relieve pathological stagnation and regulate the functional activities of meridians and internal organs $(19,22)$. Moreover, exercisers can achieve the efficiency of "body and mind relaxation" and "harmony of nature and man", thereby experiencing emotional stability, improving strength and health (23-25). Due to the 
remarkable effect of Qigong in improving physical and mental health and the ease of learning, it is suitable for almost anyone of any age and physical condition. More importantly, it is not be restricted by time, place and equipment(26, 27). In comparision with other traditional physical exercises, Qigong is clinically found effective in the treatment of CFS (28). In addition. relevant studies have supported the efficacy of Qigong threrapy in term of fatigue, sleep disorders and depression symptoms $(7,29,30)$.

Prolong life with nine turn method (PLWNT) is a type of Qigong with external energy to strengthen the limbs, internal energy to reconcile the viscera, and to smooth the qi and blood that circulated by a centenarian named Kai Fang in the Qing Dynasty. It has been written into the college textbook of Tuina and Qigong, which includes eight kinds of massage manipulations on the abdomen and a kind of upper body shaking (31). The exercise process focuses on practicing muscles, bones and skin externally, and training the spirit, breath and energy internally (32). Several randomized controlled trials have proved the efficacy of abdominal massage manipulation therapy in the treatment of patients with chronic fatigue (33), chronic insomnia(34), functional dyspepsia(35), depression(36) or spinal pain(37). These were proved to be effective of abdominal massage manipulation therapy in preventing and treating the risk factors of symptoms in terms of fatigue, cognitive function and sleep quality by stimulating the smooth muscles of the abdomen and intestines, regulating abdominal blood flow and lymphatic system function(38-40). Specifically, abdominal massage manipulation therapy can relieve muscle tension and regulate mood through electromyographic signals, neuromuscular synthesis, and nerve rhythm, thereby significantly relieving sleep disorders, fatigue and depression symptoms of CFS patients, and then further improving their life quality(41). Our recently published protocol for this project predicts that PLWNT Qigong exercise can improve fatigue, sleep disoders and depression in CFS patients (32). Therefore, this study compares the effects of PLWNT and CBT therapies to verify the effective methods to improve fatigue, sleep, anxiety and quality of life in CFS patients.

The purpose of this study is to evaluate the improvement of PLWNT on fatigue, sleep, and depression symptoms of CFS patients and the linear correlation between the fatigue and sleep, and depression symptoms.

\section{Materials \& Methods}

\section{1 study design}

The present study is a randomized controlled trial with three parallel groups: HC group and two treatment groups including PLWNT and CBT groups. All participant were recruited from December, 2018 to September, 2019 at the Shanghai University of Traditional Chinese Medicine and Yueyang Hospital of Integrated Traditional Chinese and Western Medicine in Shanghai, China. The statistician who did not participate in the recruitment randomly divided the eligible CFS patients into two groups using sealed envelope randomization. by a computer software (Strategic Applications Software, version 9.1.3; SAS Institute Inc., Cary, NC, USA) to create a random number table, and then compiled a set of sealed envelopes on the basis of the random sequence, put the patient's information, treatment method, time and location in an opaque envelope according to random numbers. Finally hand it over to the research team. The study was conducted in accordance with the Declaration of Helsinki and the International Code of Ethics for Biomedical Research Involving Human Subjects, which was approved by the Ethics Committee of Yueyang Hospital of Integrated Traditional Chinese and Western Medicine (Ethics Approval Number: 2018-043), and registered on 4 November 2018 in the American Clinical Trial Registry (Registration Number:NCT03496961).

\subsection{Sample size calculation}

According to our recently published protocol (32), the efficacy of the PLWNT group is assumed to be better than that of the CBT group. With reference to the studies on the efficacy of CFS on the FSS scale $(42,43)$, it is calculated that the final difference between the two three groups of FSS average scores is 0.915 and the standard deviation is 1.147. The conservative comparison method Bonferroni was used and the sample size of this trial was calculated using the following formula:

$n=\frac{2 \times\left(Z_{\alpha / 4}+Z_{\beta}\right)^{2} \times \sigma^{2}}{\delta^{2}}$

$=\frac{2 \times(2.2414+1.282)^{2} \times 1.1479^{2}}{0.915^{2}}=39.08 \approx 40$

Taking into account the allowable $10 \%$ dropout rate, the sample size of each group in this experiment is 45 . Therefore, this randomized controlled trial (RCT) needs to recruit 135 participants in total.

\subsection{Subjects}

135 participants were recruited on WeChat (Tencent co., LTD, China) or posters at the Shanghai University of Traditional Chinese Medicine and Yueyang Hospital of Integrated Traditional Chinese and Western Medicine in Shanghai, China. Hospitalized patients were also included with a preliminary diagnosis of CFS, according to the latest Revise Guidelines for Treatment of Chronic Fatigue Syndrome in 2021(44).

The inclusion criteria were as follows: (1) age between 20 and 60 years; no gender requirement; (2) severe chronic fatigue of no less than 6 months, unexplained after clinical evaluation; fatigue not caused by work performed during the trial and unable to be alleviated after rest; and (3) at least four of these eight symptoms (memory or concentration decline, failure to regain energy after sleep, sore throat, headache, lymph node tenderness, muscle pain, multiple joint pain, and myalgia after exertion for more than 24 hours).

The exclusion criteria were as follows: severe cardiovascular and cerebrovascular diseases, endocrine system diseases, motor system diseases, autoimmune diseases, infectious diseases, use of medications that may affect the judgment of the results. 
Patients who meet the inclusion and exclusion criteria would have a baseline measurement (T0) and be randomly assigned to the PLWNT group or CBT group. The clinical scale evaluation would be conducted at the end of the intervention ( $T 1$ ). All of the patients involved in the study would be required to sign an informed consent. A more detailed fundamental information of CFS patients is provided in our previously published protocol(32).

\subsection{Intervention}

\section{PLWNT group}

The PLWNT intervention program and operating standards refer to the Chinese general higher TCM compiled college textbook of Tuina and Qigong. Experienced Qigong teachers at Shanghai University of Traditional Chinese Medicine, who have been teaching Qigong for at least 5 years, were in charge of the supervision of the exercise and corrected the exercise posture during the entire intervention period for one hour every Sunday. The first 10 minutes is used to perform stretching and relaxation exercises, as well as movement introductions and demonstrations. In addition, precautions were mentioned and participants' questions were answered. The subsequent 20 minutes is for individual guidance and correction of actions. Finally, all of the participants practiced PLWNT for 30 minutes together. For the remaining 6 days of the week, all the participants had to practice by themselves for 30 minutes at 6 o'clock every day at home, which is under the supervision of one of the directors. Their practice videos were required to be posted in a Wechat group including all participants. If some of the participants found it inconvenient, videos can be sent privately. All participants are asked to writing down their feelings in the practice recording notebook after every exercise. The entire practice process lasted for 12 weeks. The content of PLWNT Qigong intervention was the same as in our previous research(32). The nine specific forms of manipulations are shown in Figure 1.

Step 1. Preparatory position.

First, relax the whole body, concentrate your thoughts, breathe evenly, put your tongue against the upper jaw, hold your dantian with your mind, and operate step by step

Step 2. PLWNT's first eight types of abdominal massage

1. Press the Danzhong acupoint (under the xiphoid process) with the middle three fingers in both hands, make a circle 21 times from the left, within 3 minutes.

2. With three fingers of both hands, rub down from Danzhong acupoint and move to the pubic symphysis below the umbilicus, repeated 21 times, within 3 minutes.

3. With three fingers in both hands, rub up from the pubic symphysis from two sides back to Danzhong acupoint until the hands were handed over, repeated 21 times, within 3 minutes.

4. With three fingers of both hands, push down from Danzhong acupoint and push it straight to the pubic symphysis, repeated 21 times within 3 minutes.

5. Rub the abdomen with the right hand from the left 21 times within 3 minutes.

6. Rub the abdomen with the left hand from the right 21 times, within 3 minutes.

7. Place the left hand on the left side of the lower waist and kidney, with the thumb forward, and the four fingers supporting the back, gently pinch it; With three fingers on the right hand, push straight from the bottom of the left breast to the groin, repeated 21 times in 3 minutes.

8. Place the right hand on the right side of the lower waist and kidney, with the thumb forward, and the four fingers supporting the back, gently pinch it; With three fingers on the left hand, push straight from the bottom of the right breast to the groin, repeated 21 times in 3 minutes.

Step 3. Seated rocking method

Sit cross-legged, hold your hands up slightly, and press the hands on your knees. The toes of both feet are slightly bent. Revolve the upper body clockwise 21 times and then revolve it counterclockwise 21 times.

\section{CBT group}

Qualified CBT therapists [e.g., diploma in CBT, or other professionally accredited qualifications involving CBT as a major part of training (e.g., a clinical or counseling psychologist degree)] were invited to conduct CBT by giving lectures or psychological consultations on the prevention and treatment of CFS for one hour, once a week. For the remaining 6 days of the week, all the participants are required to listen to lectures on Wechat for 30 minutes every day. If some of the participants found it inconvenient, they are allowed to learn by their own with the given powerpoints. Each participant was asked to write down their feelings in the practice recording notebook after each online study to ensure that the other conditions were the same as those of the PLWNT group. The entire practice process lasted for 12 weeks. The detailed information is available in the previously published protocol(32).

\section{$\mathrm{HC}$ group}

Healthy participants did not receive any treatment after the beginning of the study and maintained their daily lives. However, it is necessary to receive a doctor's health review on a regular basis and fill in a health status form every week. If participants have any health problems during the progress of the study, you need to contact the doctor immediately to ensure that the data is scientific and reliable.

\subsection{Outcomes}

Outcomes evaluation included the basic characteristics of personal information, the detection of peripheral blood of CFS, the quality of sleep, mental and physical fatigue, anxiety and depression symptoms. Evaluate the patient's basic information at baseline, use the relevant self-assessment scale and peripheral 
blood concentration to evaluate the primary and secondary outcomes after 12 weeks of intervention, including the Multidimensional Fatigue Inventory-20 (MFI-20), Pittsburgh Sleep Quality Index (PSQI) , and Hospital Anxiety and Depression Scale (HADS).

\section{Primary outcomes}

\section{MFI-20}

Multi-dimensional Fatigue Inventory-20 (MFI-20) is widely used for CFS measurement of mental and physical fatigue (45), a total of 20 items, including five dimensions of general fatigue, physical fatigue, mental fatigue, reduced activity, and reduced motivation. Each item can be scored on a scale of $0-5$. The total score was 0-100 points. The higher the score is, the severer the fatigue is.

\section{Secondary outcomes}

\section{Overall efficacy evaluation}

The overall efficacy evaluation was formulated with reference to the efficacy standard established by "Discussion on the Curative Effect Standards for Diagnosis and Treatment of Chronic Fatigue Syndrome" $(46,47)$ and combined with the MFI-20 score. Full recovery was defined as the complete disappearance of the main clinical symptoms and concurrent symptoms, and a reduction of MFI-20 score by more than $95 \%$. Markedly effective is defined as the disappearance of more than $2 / 3$ main clinical symptoms and concurrent symptoms, and a reduction of MFI- 20 score by more than $70 \%$. Effective as the disappearance of more than $1 / 3$ main clinical symptoms and concurrent symptoms, and a reduction of MFI- 20 score by more than $30 \%$. Ineffectiveness as the disappearance of the main clinical symptoms and concurrent symptoms $<1 / 3$ or no improvement, and a reduction of MFI- 20 score by less than $30 \%$. The total effective rate is the sum of the recovery rate, the markedly effective rate, and the effective rate.

\section{PSQI}

PSQI is a self-assessment questionnaire used to evaluate sleep quality. The scale consists of 24 items, with 19 self-reported items and 5 additional items rated by the director but not scored. The 19 items belong to one to the seven subcategories: subjective sleep quality, sleep latency, sleep duration, habitual sleep efficiency, sleep disturbances, use of sleeping medication, and daytime dysfunction(48). The score range for each dimension is from 0 to 3 points, and the total score range is 0-21 points. The higher the score is, the worse the sleep quality is.

\section{HADS}

Hospital Anxiety and Depression Scale (HADS) is used to evaluate the degree of anxiety and depression of patients. The scale consists of 14 items, of which 7 items assess anxiety and the other 7 items assess depression. The score for each item, ranges from 0 point (nothing at all) to 3 points (the extreme form of each symptom)(49). The higher the score is, the severer the degree of anxiety and depression is.

\subsection{Correlations}

Possible determinants are investigated of the total score of fatigue, sleep quality, and anxiety and depression at the end of treatment (T1). The linear relationship among them has been further explored.

\subsection{Adverse event}

We did our best to prevent and treat the damage that may be caused by this research. If there was an adverse event in the clinical trial, including any discomfort, or new changes in the condition, or any unexpected situation, it would be sent to the nearby Yueyang Hospital of Integrated Traditional Chinese and Western Medicine for review in time, and the Medical Expert Committee determined whether it was related to treatment. The expert committee has determined that the adverse event was related to treatment, and the cost of treatment and corresponding financial compensation was provided for the damage related to the trial. All unexpected and unexpected reactions reported by each participant was recorded on the adverse event report form. Follow up all adverse events until resolved. Using 1 to 4 to indicate continuous increase in severity degree between PLWNT treatment and adverse events( $1=$ Definitely not related, $2=$ Probably not related, $3=$ Probably related, $4=$ Definitely related).

\subsection{Statistical analysis}

SPSS 25.0 (SPSS version 25.0, SPSS Inc. Chicago, IL, USA) was used for statistical analysis. For measurement data such as age and scale score, the average value \pm standard deviation $(S)$ was used. For measurement data conforming to normal distribution and homogeneity of variance test, One-way analysis of variance was used for the comparison between the three groups, two independent sample T test was used for comparison between groups. For non-normally distributed measurement data, Kruskal-Wallis nonparametric test was used for comparison between the three groups, two independent samples of MannWhitney non-parametric test were used between groups, and $\mathrm{P}<0.05$ indicated that the difference was statistically significant. The Spearman correlation analysis was used to study the possible relationship between the MFI-20 and the clinical features of the PSQI and HADS scale scores.

\section{Results}

A total of 135 participants who met the criteria were recruited in this study. They were randomly divided into PLWNT group, CBT group and HC group, with 45 people in each group. Among them, one case in the CBT group withdrew due to shoulder fracture, which was a lost case. In the end, 134 participants completed the clinical observation of the entire treatment. The process is shown in the flowchart (Figure 2). 


\subsection{Demographic And Clinical Characteristics}

The baseline characteristics of the PLWNT group and CBT group are shown in Table 1, which shows that no difference is shown between the two groups, such as male-female ratio, age, height, education in three groups. Fatigue, sleep and depression scale between PLWNT and CBT group were comparable, with the $\mathrm{P}$ values are $0.513,0.123$ and 0.855 , respectively $(P>0.05)$, the difference between the treatment groups (PLWNT and CBT group) and the HC group at baseline was statistically significant $(P<0.05)$. These are indicated that the baseline is relatively uniform. The participants were between 20 and 50 years old. Most of them are female students in high schools or universities. Nearly half of them are married. They joined in the trial mainly due to the referral of the hospital. In terms of MFI-20, PSQI and HADS scales, patients who score higher suffer severer symptoms of fatigue, sleep disorders and depression.

Table 1

Demographic and clinical characteristics of the patients.

\begin{tabular}{|c|c|c|c|c|}
\hline & $\begin{array}{l}\text { PLWNT } \\
(n=45)\end{array}$ & $\begin{array}{l}\text { Control } \\
(n=44)\end{array}$ & $\begin{array}{l}\mathrm{HC} \\
(n=45)\end{array}$ & $P$ vaule \\
\hline Age (year) & $37.943 \pm 11.344$ & $37.343 \pm 9.864$ & $38.257 \pm 11.521$ & 0.935 \\
\hline Weight (kg) & $59.804 \pm 10.893$ & $61.943 \pm 12.061$ & $58.985 \pm 12.832$ & 0.557 \\
\hline Height (cm) & $163.514 \pm 6.679$ & $165.000 \pm 7.376$ & $166.629 \pm 8.748$ & 0.209 \\
\hline Gender(M\F) & $17 \backslash 28$ & $19 \backslash 25$ & $19 \backslash 26$ & - \\
\hline Education(year) & $11.823 \pm 3.25$ & $11.232 \pm 2.86$ & $12.016 \pm 2.821$ & - \\
\hline Married & 21 & 20 & 19 & - \\
\hline Single & 23 & 22 & 25 & - \\
\hline Divorce & 1 & 2 & 1 & - \\
\hline Clinical score & $10.556 \pm 2.896$ & $10.932 \pm 2.872$ & $4.381 \pm 2.279 \# \$$ & 0.000 \\
\hline MFI-20 & $6.756 \pm 3.523$ & $7.705 \pm 2.953$ & $3.401 \pm 2.016 \# \$$ & 0.000 \\
\hline PSQI & $6.844 \pm 4.033$ & $6.705 \pm 3.927$ & $2.640 \pm 2.604 \# \$$ & 0.000 \\
\hline \multicolumn{5}{|l|}{ HADS } \\
\hline \multicolumn{5}{|c|}{ \# Indicates that the difference between the PLWNT and HC group is statistically significant $(p<0.05)$} \\
\hline$\$$ indicates that & rence between & $\mathrm{T}$ and $\mathrm{HC}$ grol & tistically signi & \\
\hline
\end{tabular}

\subsection{Outcome Measurements}

Table 2suggests the total effective rate of PLWNT and CBT group. the results showed total effective rate of the PLWNT group was $62.22 \%$, in which 0 case is cured, 7 cases are markedly effective, 21 cases are effective and 17 cases are no effective. The total effective rate of the CBT group was $50 \%$, in which 0 case is cured, 0 cases are markedly effective, 22 cases are effective and 22cases are no effective. There was a significant difference between the two groups $(\mathrm{P}<0.05)$, indicating that the effectiveness of PLWNT group in the treatment of CFS was higher than that of CBT group.

Table 2

Efficacy evaluation of PLWNT group and CBT group after 12 weeks of intervention (Cases/effective rate)

\begin{tabular}{|lllllll|}
\hline Group & Cases & Cases/cured(\%) & Cases/markedly effective(\%) & Cases/ effective(\%) & Cases/ no effective(\%) & Total effective(\%) \\
\hline PLWNT & 45 & $0 / 0$ & $7 / 15.56$ & $21 / 46.67$ & $17 / 37.78$ & 62.22 \\
CBT & 44 & $0 / 0$ & $0 / 0$ & $22 / 50$ & $22 / 50$ & 50 \\
\hline
\end{tabular}

Table 3 shows the changes in the primary and secondary score of MFI-20, PSQI, and HADS scales from T0 to T1 in three groups. The results indicat that the primary outcome of MFI-20 significantly decreased after 12 weeks of intervention in the PLWNT and CBT groups, which shows statistical significant difference $(p<0.001)$. Thus, conditions have improved in terms of general fatigue, physical fatigue, reduced activity, reduced motication and mental fatigue. As for the secondary outcomes, the overall scores of PSQI and HADS scales have improved comparing T1 to T0 in the PLWNT and CBT groups. The comparison of HADS within the groups showed statistical significance after the PLWNT and CBT intervention. According to the results of PSQI Scale, significantly improved are the overall sleep quality, subject sleep quality, sleep latency, sleep duration, sleep disturbance and daytime dysfunction after intervention ( $p<$ 0.05). However, in terms of habitual sleep efficiency and sleep medicine using, there was no statistical significant difference between the groups ( $p>0.05$ ). The total scores and the average value in each score of the changes of MFI-20, PSQI and HAS scales in the PLWNT group were greater than those in the CBT group although there was no statistical significance between the groups. In additional, the changes in the PLWNT and CBT groups were higher than those in the HC group except for habitual sleep efficiency and sleep medicine using $(p<0.05)$. 
Table 3 - shows the changes primary and secondary outcomes in fatigue, sleep, anxiety and depression scores measured by MFI-20, PSQI, and HADS scales from T0 to T1.

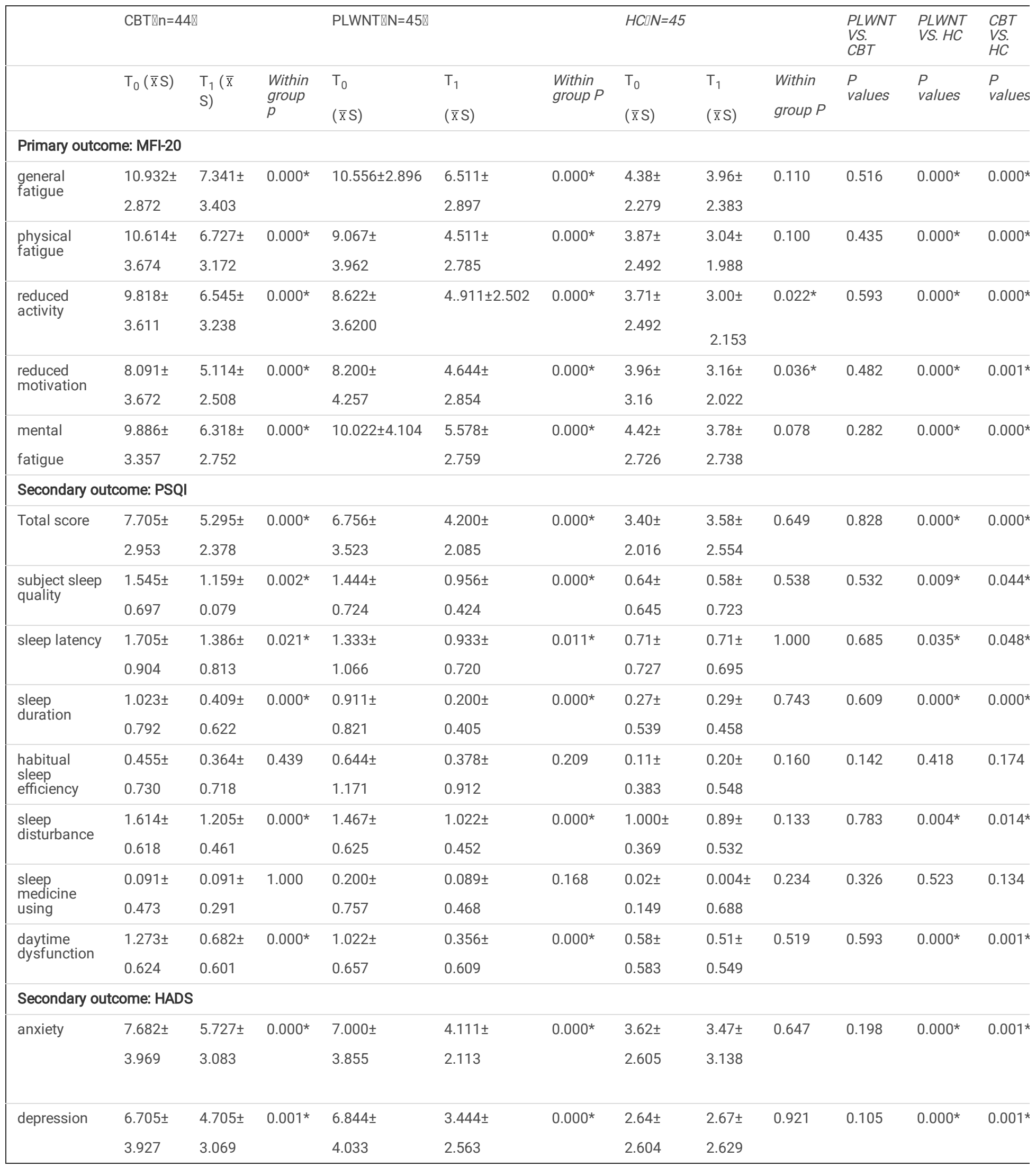

\subsection{Correlations}


Fatigue was the primary result of the study, the treatment groups performed additional correlation analysis between the primary and secondary outcomes. The Pearson correlation coefficient was used to explore the relationship between fatigue and sleep quality as well as fatigue and anxiety. For PLWNT group, fatigue level is positively correlated with sleep quality $(r=0.315)$ and anxiety level $(r=0.333)(p<0.05)$. However, for CBT group, fatigue level was only related to anxiety level $(r=0.332)(p<0.05)$, and shows no correlation with sleep quality $(p>0.05)$. The specific results were shown in Figure 3 .

\subsection{Adverse Event}

Table 4displays a total of 6 participants who reported 6 adverse events in our study. Among them, two cases were determined to be definitely or probably related to the exercises with mild symtoms, which were caused by improper massage manipulations on the abdomen. Except for one patient who chose to quit due to the shoulder fracture, all others continued the treatment after the adverse events had been properly dealt with.

Table 4

Adverse event

\begin{tabular}{|c|c|c|c|c|c|c|c|}
\hline symptom & $\begin{array}{l}\text { Patients } \\
\text { numbers }\end{array}$ & Start date & End date & Relationship & Treatment & $\begin{array}{l}\text { Action related to } \\
\text { intervention }\end{array}$ & Outcome \\
\hline Leg pain & 1 & 20190224 & 20190303 & Probably & None & No change & Cured \\
\hline $\begin{array}{l}\text { Shoulder } \\
\text { fracture }\end{array}$ & 1 & 20190309 & 20190310 & $\begin{array}{l}\text { Definitely } \\
\text { not }\end{array}$ & Medication & Withdraw & Unknown \\
\hline Dizziness & 1 & 20190512 & 20190512 & $\begin{array}{l}\text { Probably } \\
\text { not }\end{array}$ & $\begin{array}{l}\text { Measure blood pressure and eat } \\
\text { breakfast }\end{array}$ & No change & Cured \\
\hline $\begin{array}{l}\text { Left thumb } \\
\text { pain }\end{array}$ & 1 & 20190721 & 20190804 & $\begin{array}{l}\text { Definitely } \\
\text { not }\end{array}$ & None & No change & Cured \\
\hline Diarrhea & 1 & 20190811 & 20190812 & $\begin{array}{l}\text { Probably } \\
\text { not }\end{array}$ & Catch cold & No change & Cured \\
\hline Chest pain & 1 & 20190815 & 20190829 & Definitely & Relieve acupoint stimulation & No change & Cured \\
\hline
\end{tabular}

\section{Discussion}

This study aims to evaluate the efficacy of PLWNT on fatigue, sleep disorders, and depression symptoms of CFS patients as well as the linear correlation between fatigue and sleep quality as well as fatigue and depression level. As the results show, significantly improved were the levels of fatigue, anxiety and depression as well as sleep quality of CFS patients after PLWNT and CBT intervention. Particularly, the self-reported sleep time increased to the minimum of 7 hours after PLWNT intervention, which was recommended in many guidelines. However, no improvement was reported from $T_{0}$ to $T_{1}$ in terms of the habitual sleep efficiency and sleep medicine using. These findings must be interpreted with caution, for the difference is not statistically significant in the levels of fatigue, anxiety and depression as well as sleep quality between the PLWNT group and the CBT group, due to the relatively small between-group effect size. Neverthless, the overall efficacy and post-test scores of the PLWNT group are better than those of the CBT group, possibly due to the milder clinical manifestations of CFS at baseline.

CFS is characterized by long-term and unexplainable fatigue(50), which can be well treated by PLWNT qigong. Previous studies have disclosed the efficacy of moxibustion, auricular acupoint pressing, Chinese medicine and other therapies on the alleviation of fatigue symptoms in CFS patients(51-53). Patients who suffer from fatigue cannot carry out or maintain a certain intensity of physiological activity. If not treated properly in time, the symptoms would deteriorate into chronic fatigue in a certain degree $(54,55)$. Studies had confirmed that the scavenging ability of oxygen free radicals decreases when the body was fatigued, and if the free radicals in the body cannot be eliminated in time, fatigue would be worsened(56, 57). PLWNT Qigong is an effective treatment of CFS, including eight kinds of massage manipulations on the abdomen and a kind of upper body shaking method. The working mechanism is to make the whole body skeletal muscles, especially of the upper limb, in a state of relaxing limb activity, enhance the body's antioxidant enzyme activity, remove oxygen free radicals and stabilize the body environment(58), which has been reported in the previous study(59) that traditional Qigong exercises can increase the activity of diaphragm and abdominal muscles, strengthen peripheral skeletal muscle function and improve fatigue symptoms. This was the main reason for the improvement of reduced activity and reduced motivation in MFI-20 of CFS patients after PLWNT intervention in this study. In addition, patients with CFS fatigue often have problems with immune dysfunction may be one of the causes of fatigue $(60,61)$. Abdominal massage manipulations can enhance the body's immunity by clearing the blood lactic acid after fatigue, improving the patient's autonomic nerve function and making the sports center in a benign state of excitement (62). This may be the main reason why PLWNT can effectively relieve mental fatigue and physical fatigue.

The overall evluation of sleep quality, subjective sleep quality, sleep latency, sleep duration, sleep disturbance and daytime dysfunction of CFS patients were significantly improved after PLWNT intervention, which was consistent with the previous study result on Baduanjin Qigong. In the previous study, Baduanjin Qigong improved the sleep latency and sleep duration of CFS patients, but did not improve subjective sleep quality and sleep disturbance(63). In this study, the better effect of PLWNT Qigong on the multi-dimensional improvement of sleep quality may also be related to the abdomen massage manipulations. Studies pointed out that abdomen massage manipulations can regulate nerve conduction connections through Brain-Gut Axis (BGA), stimulate nerve conduction pathways and nerve-emotional pathways through internal organs, strengthen the connection between the abdomen and cranial nerves to regulate fatigue and sleep in CFS patients $(64,65)$. It was worth noting that no improvement was shown in terms of the habitual sleep efficiency and the use of sleep drugs of the participants in this study. It was consistent with several previous studies on CFS, which reported that CFS patients were more likely to be awakened and had a

Page $8 / 14$ 
longer sleep latency than healthy people $(66,67)$. Therefore, it is speculated that the lack of improvement in habitual sleep efficiency may be caused by sleep interruption or a prolonged sleep latency, leading to the increasing time in bed. On the other hand, the exclusion criteria excluding all subjects taking medications may be the reason for the insignificant difference in the use of sleep drugs.

The anxiety and depression levels of CFS patients decreased significantly after PLWNT intervention. It has been reported that the use of Baduanjin Qigong exercise has antidepressant effects on women of CFS-like diseases(30). The limbic system of the human brain is responsible for emotion regulation. Therefore, anxiety and depression may be related to the dysfunction of the brain network connecting the limbic system and cortex(68). In some studies, antidepressants are used to increase serotonin levels and reduced cortisol secretion in the brain to deal with anxiety and depression of CFS patients, but the drugs have side effects such as headache, sleep disturbance, cardiovascular function changes and bone loss (23). It is reported that rubbing the abdomen can increase serotonin and endorphins to activate the spinal cord and subcortical nucleus activity to reduce the level of anxiety and depression(69). Thus, PLWNT may be used to relieve anxiety and depression symptoms through abdominal pressing and rubbing.

The improvement of fatigue, sleep and anxiety symptom improvement was directly related to the amount of qigong exercises, which was confirmed by previous studies on other types of Qigong $(70,71)$. In this study, the Person correlation coefficient is used to discover that fatigue level is positively correlated with anxiety level and sleep quality. A new study conducted by Russell et al. reported to have similar results, in which the sleep quality of 27 adult CFS patients(72) is evaluated by sleep-wake diary and activity recorder, revealing that sleep disorder induces a more serious level of fatigue due to hypothalamicpituitary-adrenal (HPA). Similarly, in the case of perception of external stress and fatigue, HPA is activated and overactive, and a large amount of glucocorticoids will be released into the body to perceive anxiety(73). This shows that fatigue was closely related to sleep and anxiety symptoms, maybe we need to increase the time of PLWNT exercise, or turn it into a daily exercise to relieve fatigue from exercise and gain more benefits.

This study did not report adverse events or withdrawals due to exercises. This shows that PLWNT exercises guided by professionals can be safely used for CFS patients. In general, our research indicates that PLWNT can provide an alternative and more acceptable form of exercise for CFS patients. This has important health care significance for CFS patients who may not be able to perform traditional exercises due to physical limitations or comorbidities. PLWNT is a mild, low-intensity exercise, usually accepted by CFS patients, and it is an advantageous method for them to comply with the World Health Organization's physical activity recommendations.

There are some limitations in the study. First of all, our participants were only recruited according to the self-reported scales. They did not receive a comprehensive physical and mental status check, due to which some patients may not fully meet the CFS inclusion criteria. However, by limiting the age of participants to 20-60 years old, the possibility of CFS caused by medical and mental conditions is reduced. Second, the experimental design itself had potential limitations. Ideally, participants should be kept unknown about the intervention. However, in fact, it is difficult to achieve for non-drug trials. Therefore, they may have high expectations for the effectiveness of the treatment, which may exaggerate the results. Third, patients over 60 years old are excluded in this study. Therefore, the results should not be generalized to the elderly over 60 years old. In addition, there were other non-specific factors, such as stretching exercises, participants' personal attention and social support, which may help improve the results. Future research will be done with interventions controlling all non-specific factors, so as to better understand the specific efficacy of PLWNT. Despite these limitations, this study is the first large-scale randomized controlled trial to prove the beneficial effects of PLWNT on CFS. Another obvious advantage is that this study reports all the adverse events in detail. We described the adverse symptoms, the time of appearance and disappearance, the relationship between the adverse events and the trial, and the related treatments and results. During the clinical trial, only 1 out of 90 participants quit due to an adverse event. The safety of Qigong treatment has been reported before and has been confirmed in the study(74-76).

\section{5.conclusion}

In summary, PLWNT has a positive effect in the treatment of fatigue, sleep disorder, anxiety and depression symptoms of CFS patients, and fatigue level is positively correlated with sleep quality as well as anxiety and depression level. PLWNT can be considered as a treatment option for CFS patients, but a more rigorous research is needed to provide clear evidences. Future studies will be carries out in a larger sample size for further in-depth research to determine the effective frequency and intensity of PLWNT Qigong intervention in the treatment of CFS. This study has significant translational significance because our findings will support the inclusion of PLWNT in the global physical activity guidelines for CFS patients.

\section{Abbreviations}

Chronic fatigue syndrome (CFS); Prolong life with nine turn method (PLWNT); International Association of Chronic Fatigue Syndrome (IACFS); cognitive behavioral therapy (CBT); traditional Chinese medicine (TCM); Multidimensional Fatigue Inventory-20 (MFI-20); Pittsburgh Sleep Quality Index (PSQI); Hospital Anxiety and Depression Scale (HADS); hypothalamic-pituitary-adrenal (HPA).

\section{Declarations}

\section{Ethics approval and consent to participate}

The study was approved by the Ethics Committee of Yueyang Hospital of Integrated Traditional Chinese and Western Medicine (Ethics Approval Number: 2018-043), and registered on 4 November 2018 in the American Clinical Trial Registry (Registration Number:NCT03496961),https://register.clinicaltrials.gov/prs/app/action/SelectProtocol?sid=S0007UWE\&selectaction=Edit\&uid=U00040E0\&ts=2\&cx=hs5jvd.

\section{Consent for publication}


All authors agreed to publish this article.

\section{Availability of data and materials}

All data generated or analysed during this study are included in this published article.

\section{Competing interests}

The authors declare that they have no competing interests.

\section{Funding}

This work was supported by the National Natural Science Foundation of China under Grant 81774443, National Natural Science Foundation of China under Grant 82105038, National Natural Science Foundation of China under Grant 873235 and the National Key Technology R\&D Program of China Grant numbers 2017 YFC1703301.

\section{Authors' Contributorship}

The project was conceived and designed by all the authors. F.F.X., and C.G. performed the search and meta-analysis. F.Y. and Y.L.Y. contributed to data analysis and interpretation F.F.X., the manuscript draft that was revised by all co-authors. All authors approved the final version of the manuscript and agree to be accountable for all aspects of the work.

\section{Acknowledgements}

We are grateful to Guanwu Li for advice relating to the analysis and thanks are due to Ruiping Wang for guidance. We also would like to thank Professor Min Fang from the University of shanghai university of traditional Chinese medicine for proofreading the manuscript.

\section{References}

1. Mandarano AH, Maya J, Giloteaux L, et al. Myalgic encephalomyelitis/chronic fatigue syndrome patients exhibit altered T cell metabolism and cytokine associations. J Clin Investig. 2020;130(3):1491-505.

2. Lim EJ, Ahn YC, Jang ES, et al. Systematic review and meta-analysis of the prevalence of chronic fatigue syndrome/myalgic encephalomyelitis (CFS/ME). Journal of translational medicine. 2020;18(1):100.

3. Global regional. national burden of multiple sclerosis. 1990-2016: a systematic analysis for the Global Burden of Disease Study 2016. Lancet Neurol. 2019;18(3):269-85.

4. Díaz-Delgado Peñas R, Gutiérrez Rivas E, Palacín Delgado C, Rivera Redondo J, Ramón Giménez JR. Chronic fatigue syndrome: aetiology, diagnosis and treatment. BMC Psychiatry. 2009 Oct 23;9 Suppl 1(Suppl 1):S1.

5. Mariman AN, Vogelaers DP, Tobback E, et al. Sleep in the chronic fatigue syndrome. Sleep medicine reviews. 2013;17(3):193-9.

6. Faro M, Sàez-Francás N, Castro-Marrero J, et al. Gender differences in chronic fatigue syndrome. Reumatologia clinica. 2016;12(2):72-7.

7. Chan JSM, Ng SM, Yuen LP, et al. Qigong exercise for chronic fatigue syndrome. International review of neurobiology. 2019;147:121-53.

8. Taylor RR. Quality of life and symptom severity for individuals with chronic fatigue syndrome: findings from a randomized clinical trial. Am J Occup Ther. 2004;58(1):35-43.

9. Castro Sánchez AM, García López H, Fernández Sánchez M, et al. Improvement in clinical outcomes after dry needling versus myofascial release on pain pressure thresholds, quality of life, fatigue, pain intensity, quality of sleep, anxiety, and depression in patients with fibromyalgia syndrome. Disabil Rehabil. 2019;41(19):2235-46.

10. Shu Q, Wang H, Litscher D, et al. Acupuncture and Moxibustion have Different Effects on Fatigue by Regulating the Autonomic Nervous System: A Pilot Controlled Clinical Trial. Scientific reports. 2016;6:37846.

11. Vercoulen JH, Swanink CM, Galama JM, et al. The persistence of fatigue in chronic fatigue syndrome and multiple sclerosis: development of a model. $J$ Psychosom Res. 1998;45(6):507-17.

12. Havlikova E, Rosenberger J, Nagyova I, et al. Impact of fatigue on quality of life in patients with Parkinson's disease. Eur J Neurol. 2010;15(5):475-80.

13. Kia S, Choy E. Update on Treatment Guideline in Fibromyalgia Syndrome with Focus on Pharmacology. Biomedicines. 2017;5(2).

14. White PD, Goldsmith KA, Johnson AL, et al. Comparison of adaptive pacing therapy, cognitive behaviour therapy, graded exercise therapy, and specialist medical care for chronic fatigue syndrome (PACE): a randomised trial. Lancet. 2011;377(9768):823-36.

15. Wilshire $\mathrm{CE}$, Kindlon T, Courtney R, et al. Rethinking the treatment of chronic fatigue syndrome-a reanalysis and evaluation of findings from a recent major trial of graded exercise and CBT. BMC psychology. 2018;6(1):6.

16. Chambers D, Bagnall AM, Hempel S, et al. Interventions for the treatment, management and rehabilitation of patients with chronic fatigue syndrome/myalgic encephalomyelitis: an updated systematic review. J R Soc Med, 99(10), 506-520.

17. McCaffrey R, Fowler NL. Qigong practice: a pathway to health and healing. Holist Nurs Pract. 2003;17(2):110-6.

18. Li R, Chen H, Feng J, et al. Effectiveness of Traditional Chinese Exercise for Symptoms of Knee Osteoarthritis: A Systematic Review and Meta-Analysis of Randomized Controlled Trials. International journal of environmental research and public health. 2020;17(21). 
19. Matos LC, Sousa CM, Gonçalves M, et al. Qigong as a Traditional Vegetative Biofeedback Therapy: Long-Term Conditioning of Physiological Mind-Body Effects. BioMed research international. 2015;2015:531789.

20. Xiong X, Wang P, Li X, et al. Qigong for hypertension: a systematic review. Medicine. 2015;94(1):e352.

21. Park JE, Liu Y, Park T, et al. A trial for the use of qigong in the treatment of pre and mild essential hypertension: a study protocol for a randomized controlled trial. Trials. 2011;12:244.

22. Vera FM, Manzaneque JM, Rodríguez FM, et al. Acute Effects on the Counts of Innate and Adaptive Immune Response Cells After 1 Month of Taoist Qigong Practice. Int J Behav Med. 2016;23(2):198-203.

23. Tsang HW, Tsang WW, Jones AY, et al. Psycho-physical and neurophysiological effects of qigong on depressed elders with chronic illness. Aging Ment Health. 2013;17(3):336-48.

24. Vergeer I, Bennie JA, Charity MJ, et al. Participation trends in holistic movement practices: a 10-year comparison of yoga/Pilates and t'ai chi/qigong use among a national sample of 195,926 Australians. BMC Complement Altern Med. 2017;17(1):296.

25. Zheng G, Lan X, Li M, et al. The effectiveness of Tai Chi on the physical and psychological well-being of college students: a study protocol for a randomized controlled trial. Trials. 2014;15:129.

26. Yeh ML, Chung YC. A randomized controlled trial of qigong on fatigue and sleep quality for non-Hodgkin's lymphoma patients undergoing chemotherapy. European journal of oncology nursing: the official journal of European Oncology Nursing Society. 2016;23:81-6.

27. Zou L, Pan Z, Yeung A, et al. A Review Study on the Beneficial Effects of Baduanjin. Journal of alternative and complementary medicine (New York, NY). 2018;24(4):324-35.

28. Klein P. Qigong in Cancer Care: Theory, Evidence-Base, and Practice. Medicines. 2017;4(1):2-.

29. Jiao J, Russell IJ, Wang W, et al. Ba-Duan-Jin alleviates pain and fibromyalgia-related symptoms in patients with fibromyalgia: results of a randomised controlled trial. Clin Exp Rheumatol. 2019;37(6):953-62.

30. Chan JS, Li A, Ng SM, et al. Adiponectin Potentially Contributes to the Antidepressive Effects of Baduanjin Qigong Exercise in Women With Chronic Fatigue Syndrome-Like Illness. Cell Transplant. 2017;26(3):493-501.

31. Shi XWS. A Brief Explanation of prolong life with nine turn method. Wudang. 2013(5):59-.

32. Xie F, Guan C, Cheng Z, et al. Effects of the prolong life with nine turn method (Yan Nian Jiu Zhuan) Qigong on patients with chronic fatigue syndrome: study protocol for a randomized controlled trial. Annals of palliative medicine. 2020;9(5):3571-83.

33. Orhan C, Özgül S, Baran E, et al. Comparison of Connective Tissue Manipulation and Abdominal Massage Combined With Usual Care vs Usual Care Alone for Chronic Constipation: A Randomized Controlled Trial. J Manipulative Physiol Ther. 2020;43(8):768-78.

34. Zhang Y, Cong D, Liu P, et al. Study on the mechanism of regulating the hypothalamic cortical hormone releasing hormone/corticotropin releasing hormone type I receptor pathway by vibro-annular abdominal massage under the brain-intestine interaction in the treatment of insomnia. Medicine. 2021;100(19):e25854.

35. Liu M, Zhou Z, Li B, Yang Q, Liu C, Chang X. [Effect difference between acupuncture and moxibustion for functional dyspepsia: a randomized controlled trial]. Zhongguo Zhen Jiu. 2017 Sep 12;37(9):943-7.

36. Zhou YF, Wei YL, Zhang PL, Gao S, Ning GL, Zhang ZQ, Hu B, Wang DY, Yan MR, Liu WJ. [Multi-central controlled study on three-part massage therapy for treatment of insomnia of deficiency of both the heart and spleen]. Zhongguo Zhen Jiu. 2006 Jun;26(6):385-8.

37. Yang JX, Zhang JP, Yu JC, Han JX. [Treatment of cervical spondylosis by spinal balancing combined with intervention of pathway of qi: a randomized controlled study]. Zhongguo Zhen Jiu. 2013 Jul;33(7):582-6.

38. Karaaslan Y, Toprak Celenay S, Kucukdurmaz F. Comparison of Pelvic Floor Muscle Training With Connective Tissue Massage to Pelvic Floor Muscle Training Alone in Women With Overactive Bladder: A Randomized Controlled Study. J Manipulative Physiol Ther. 2021;44(4):295-306.

39. Silva CA, Motta ME. The use of abdominal muscle training, breathing exercises and abdominal massage to treat paediatric chronic functional constipation. Colorectal disease: the official journal of the Association of Coloproctology of Great Britain Ireland. 2013;15(5):e250-5.

40. Ussing A, Dahn I, Due U, et al. Efficacy of Supervised Pelvic Floor Muscle Training and Biofeedback vs Attention-Control Treatment in Adults With Fecal Incontinence. Clinical gastroenterology hepatology: the official clinical practice journal of the American Gastroenterological Association. 2019;17(11):2253-61.e4.

41. Langhorst J, Klose P, Dobos GJ, et al. Efficacy and safety of meditative movement therapies in fibromyalgia syndrome: a systematic review and metaanalysis of randomized controlled trials. Rheumatol Int. 2013;33(1):193-207.

42. Sharpe M, Hawton K, Simkin S, et al. Cognitive behaviour therapy for the chronic fatigue syndrome: a randomized controlled trial. BMJ. 1996;312(7022):22-6.

43. Cumming TB, Packer M, Kramer SF, et al. The prevalence of fatigue after stroke: A systematic review and meta-analysis. International journal of stroke: official journal of the International Stroke Society. 2016;11(9):968-77.

44. UK Moves to Revise Guidelines for Treatment of Chronic Fatigue Syndrome. The American journal of nursing. 2021 Mar 1;121(3):16.

45. Smets EM, Garssen B, Bonke B, et al. The Multidimensional Fatigue Inventory (MFI) psychometric qualities of an instrument to assess fatigue. J Psychosom Res. 1995;39(3):315-25.

46. Larun L, Brurberg KG, Odgaard-Jensen J, et al. Exercise therapy for chronic fatigue syndrome. Cochrane Database Syst Rev. 2017;4(4):CD003200.

47. Cleare AJ, Reid S, Chalder T, et al. Chronic fatigue syndrome. BMJ clinical evidence. 2015;2015. 
48. Buysse DJ, Reynolds CF 3rd, Monk TH, et al. The Pittsburgh Sleep Quality Index: a new instrument for psychiatric practice and research. Psychiatry research. 1989;28(2):193-213.

49. Bjelland I, Dahl AA, Haug TT, et al. The validity of the Hospital Anxiety and Depression Scale: An updated literature review. J Psychosom Res. 2002;52(2):0-77.

50. Clayton EW. Beyond myalgic encephalomyelitis/chronic fatigue syndrome: an IOM report on redefining an illness. Jama. 2015;313(11):1101-2.

51. Zhang F, Shen Y, Li J, et al. Auricular acupuncture for insomnia of chronic fatigue syndrome: a case report. Acupuncture in medicine: journal of the British Medical Acupuncture Society. 2020;38(5):366-8.

52. Alraek T, Lee MS, Choi TY, et al. Complementary and alternative medicine for patients with chronic fatigue syndrome: a systematic review. BMC Complement Altern Med. 2011;11:87.

53. Liu CZ, Lei B. [Effect of Tuina on oxygen free radicals metabolism in patients with chronic fatigue syndrome]. Zhongguo Zhen Jiu. 2010 Nov;30(11):9468.

54. Finsterer J, Mahjoub SZ. Fatigue in healthy and diseased individuals. Am J Hosp palliat Care. 2014;31(5):562-75.

55. Nocerino A, Nguyen A, Agrawal M, et al. Fatigue in Inflammatory Bowel Diseases: Etiologies and Management. Advances in therapy. 2020;37(1):97-112.

56. Missailidis D, Annesley SJ, Allan CY, et al. An Isolated Complex V Inefficiency and Dysregulated Mitochondrial Function in Immortalized Lymphocytes from ME/CFS Patients. International journal of molecular sciences. 2020;21(3).

57. Fluge $\varnothing$, Mella 0 , Bruland 0 , et al. Metabolic profiling indicates impaired pyruvate dehydrogenase function in myalgic encephalopathy/chronic fatigue syndrome. JCl insight. 2016;1(21):e89376.

58. Minagawa T, Domen T, Suzuki T, et al [EFFECTIVENESS OF HOCHUEKKITO (JAPANESE HERBAL MEDICINE) FOR GENERAL FATIGUE AFTER INTRODUCTION OF ENZALUTAMIDE IN THREE CASES OF CASTRATION-RESISTANT PROSTATE CANCER]. Nihon Hinyokika Gakkai zasshi The japanese journal of urology. 2019;110(2):86-91.

59. Wu W, Liu X, Liu J, et al. Effectiveness of water-based Liuzijue exercise on respiratory muscle strength and peripheral skeletal muscle function in patients with COPD. Int J Chronic Obstr Pulm Dis. 2018;13:1713-26.

60. Sheill G, Guinan E, O'Neill L, et al. Preoperative exercise to improve fitness in patients undergoing complex surgery for cancer of the lung or oesophagus (PRE-HIIT): protocol for a randomized controlled trial. BMC Cancer. 2020;20(1):321.

61. Sotzny F, Blanco J, Capelli E, et al. Myalgic Encephalomyelitis/Chronic Fatigue Syndrome - Evidence for an autoimmune disease. Autoimmun rev. 2018;17(6):601-9.

62. Chen PJ, Chou CC, Yang L, et al. Effects of Aromatherapy Massage on Pregnant Women's Stress and Immune Function: A Longitudinal, Prospective, Randomized Controlled Trial. Journal of alternative and complementary medicine (New York, NY). 2017;23(10):778-86.

63. Chan JS, Ho RT, Chung KF, et al. Qigong exercise alleviates fatigue, anxiety, and depressive symptoms, improves sleep quality, and shortens sleep latency in persons with chronic fatigue syndrome-like illness. Evidence-based complementary alternative medicine: eCAM. 2014;2014:106048.

64. Dong F, Yu H, Ma J, et al. Exploring association between gastrointestinal heat retention syndrome and recurrent respiratory tract infections in children: a prospective cohort study. BMC Complement Altern Med. 2016;16:82.

65. Beaumont A, Burton AR, Lemon J, et al. Reduced cardiac vagal modulation impacts on cognitive performance in chronic fatigue syndrome. PloS one. 2012;7(11):e49518.

66. Ohinata J, Suzuki N, Araki A, et al. Actigraphic assessment of sleep disorders in children with chronic fatigue syndrome. Brain Dev. 2008;30(5):329-33.

67. Pedersen M, Ekstedt M, Småstuen MC, et al. Sleep-wake rhythm disturbances and perceived sleep in adolescent chronic fatigue syndrome. J Sleep Res. 2017;26(5):595-601.

68. Leuchter AF, Cook IA, Hunter AM, et al. Resting-state quantitative electroencephalography reveals increased neurophysiologic connectivity in depression. PloS one. 2012;7(2):e32508.

69. Bervoets DC, Luijsterburg PA, Alessie JJ, et al. Massage therapy has short-term benefits for people with common musculoskeletal disorders compared to no treatment: a systematic review. Journal of physiotherapy. 2015;61(3):106-16.

70. Lynch M, Sawynok J, Hiew C, et al. A randomized controlled trial of qigong for fibromyalgia. Arthritis research therapy. 2012;14(4):R178.

71. Yeh SC, Chang MY. The effect of Qigong on menopausal symptoms and quality of sleep for perimenopausal women: a preliminary observational study. Journal of alternative and complementary medicine (New York, NY). 2012;18(6):567-75.

72. Russell C, Wearden AJ, Fairclough G, et al. Subjective but Not Actigraphy-Defined Sleep Predicts Next-Day Fatigue in Chronic Fatigue Syndrome: A Prospective Daily Diary Study. Sleep. 2016;39(4):937-44.

73. Kim YK, Na KS, Myint AM, et al. The role of pro-inflammatory cytokines in neuroinflammation, neurogenesis and the neuroendocrine system in major depression. Prog Neuro-psychopharmacol Biol Psychiatry. 2016;64:277-84.

74. Yu P, Li W, Li H, et al. The efficacy and safety of health qigong for anti-aging: Protocol for a systematic review and meta-analysis. Medicine. 2020;99(49):e22877.

75. Chen M, Ou L, Chen Y, et al. Effectiveness and safety of Baduanjin exercise (BDJE) on heart failure with preserved left ventricular ejection fraction (HFpEF): A protocol for systematic review and meta-analysis. Medicine. 2020;99(46):e22994.

76. Song Y, Li J, István B, et al. Current Evidence on Traditional Chinese Exercises for Quality of Life in Patients With Essential Hypertension: A Systematic Review and Meta-Analysis. Frontiers in cardiovascular medicine. 2020;7:627518. 
Figures
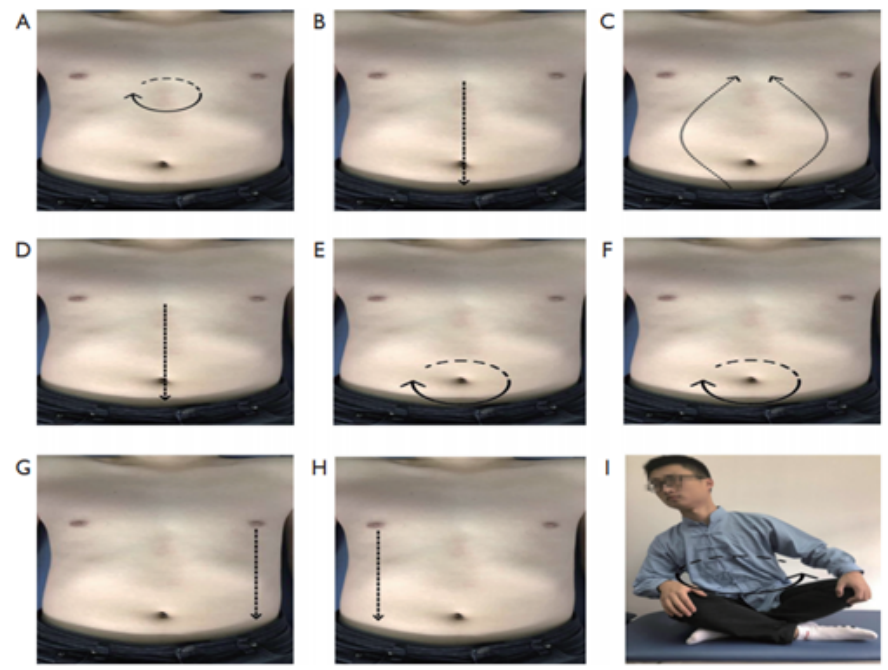

Figure 1

The postures of PLWNT. (A) Press and knead acupoint in Shanzhong. (B) Rubbing from Shanzhong Acupoint to Pubic Symphysis. (C) Rubbing from Pubic Symphysis to Shanzhong Acupoint. (D) Pushing from Shanzhong Acupoint to Pubic Symphysis. (E) The right hand massages the abdomen by the left circle. (F) The left hand massages the abdomen by the right circle. (G) Pushing with the right hand from the left breast to the groin. (H) Pushing with the left hand from the right breast to the groin. (I) Turn left and right. Every movements will be carried out 21 times. PLWNT, prolong life with nine turn method.

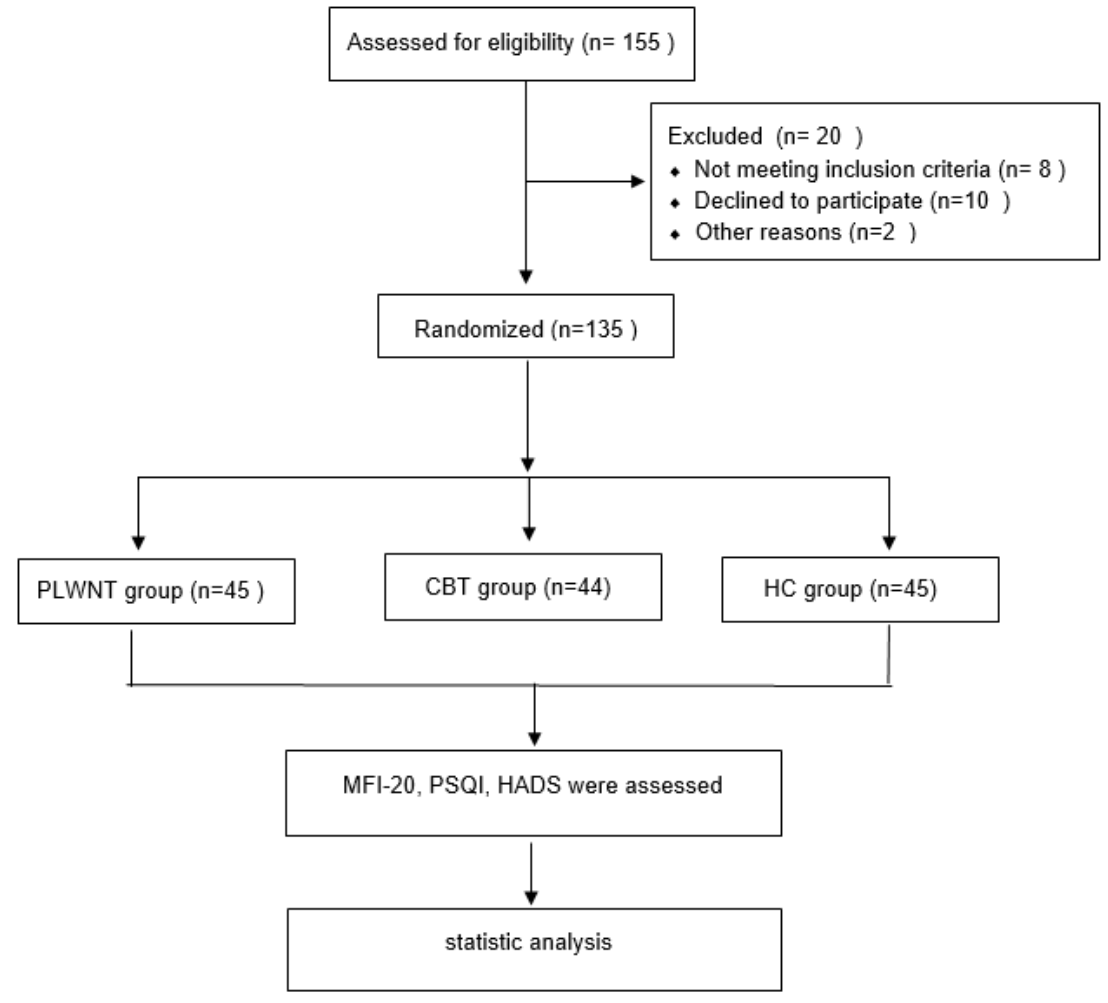

Figure 2

Recruitment flowchart. 

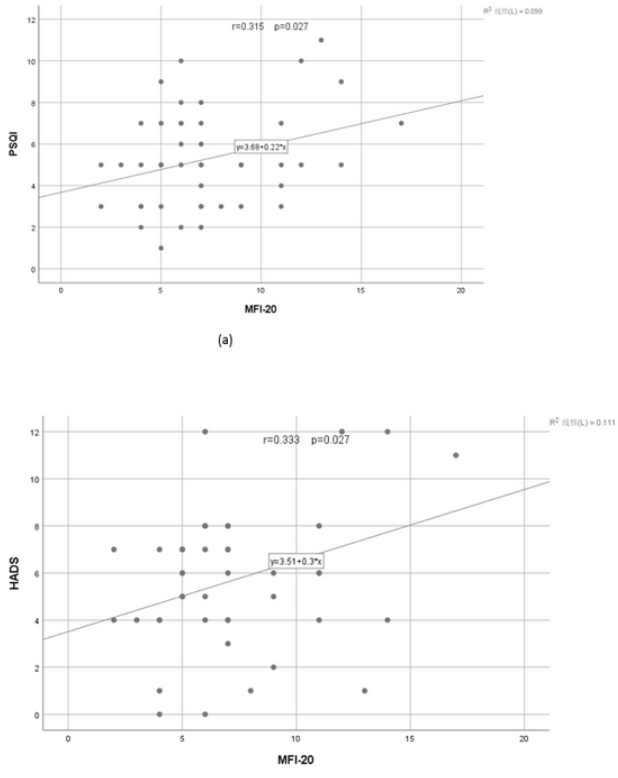

(b)

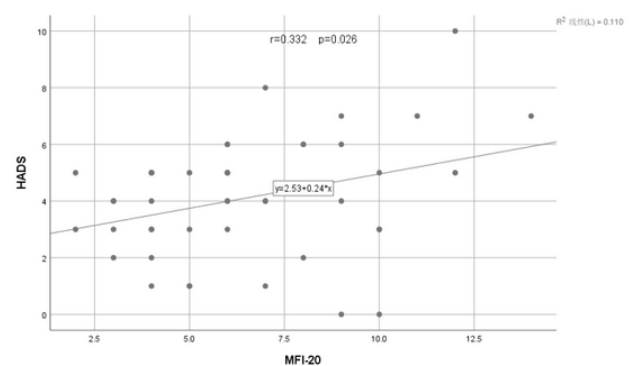

(c)

\section{Figure 3}

The linear relationship between the total score of fatigue and the total score of sleep and anxiety at the end of treatment. (a) The relationship between the total score of fatigue and the total score of sleep after PLWNT exercise. (b) The relationship between the total score of fatigue and the total score of anxiety after PLWNT exercise. (c) The relationship between the total score of fatigue and the total score of anxiety after CBT intervention. 